\title{
Appropriate indications for radiofrequency catheter ablation
}

\begin{abstract}
Most cardiologists take a very conservative approach to treatment of benign ventricular extrasystoles, usually advising no treatment or perhaps $\beta$ blockade if a patient has significant symptoms. On page 492 Gumbrielle and coworkers describe the apparently successful use of radiofrequency catheter ablation in a patient with this common clinical condition. Should these patients (or an identifiable subgroup) be referred for ablation? In considering the indications for radiofrequency catheter ablation, like those for any treatment, the efficacy and safety of the procedure need to be taken into account, in addition to the symptoms and prognosis of the condition to be treated.
\end{abstract}

\section{Efficacy and safety of radiofrequency catheter ablation for ventricular arrhythmias}

The evidence to date on catheter ablation therapy for sustained ventricular tachycardia suggests that it can be performed effectively and safely (at least in the medium term) in patients with structurally normal hearts ${ }^{1-6}$ : the site or origin of tachycardia in these patients is usually in the right ventricular outflow tract (as in the case of the patient described by Gumbrielle $e t a l$ ) or the infero-apical left ventricular septum. In patients with previous myocardial infarction or cardiomyopathy the sites of origin are much more variable and success rate is low. Procedure times are unpredictable in all these situations and frequently extend beyond 2 hours. Serious complications (thromboembolism, tamponade) are significantly more common in patients with structural heart disease but are likely to be rare in patients with "normal hearts".

\section{Effect of radiofrequency catheter ablation on symptoms and prognosis}

For catheter ablation to control symptoms effectively there must be a clear relation between the arrhythmia and the symptoms. Such a relation can be established easily in patients with recurrent sustained arrhythmias but is much more difficult in those with ventricular ectopy, because the arrhythmia is common in normal, symptom free individuals. Indeed it might be expected that, among most patients with atypical symptoms in whom only ventricular extrasystoles have been identified, the symptoms and extrasystoles will be unrelated.

Nearly all catheter ablation procedures (including accessory pathway ablations) are performed because of symptoms rather than to improve prognosis. In those cases of ventricular tachycardia that can be treated by catheter ablation it is very unlikely (and there is no evidence) that this treatment alters the prognosis in either direction. The alternative option of prophylactic medical treatment, particularly with agents of Vaughan Williams class 1 (quinidine, flecainide) may carry an adverse prognosis.

Radiofrequency ablation may be appropriate in patients with ventricular extrasystoles but only when the patient has a structurally normal heart with frequent symptoms that are clearly related to ventricular ectopy and cannot be controlled by first line antiarrhythmic agents (including $\beta$ blockade). Ideally the origin of the extrasystoles should be in the right ventricular outflow tract (left bundle branch block configuration with inferior axis). Gumbrielle and coworkers are to be congratulated for identifying such a case: it is likely to be some time before they see another.

Academic Department of Cardiology, Glenfield Hospital, Leicester LE3 $9 Q P$

CLIFFORD J GARRATT

1 Morady F, Kadish AH, DiCarlo L, et al. Long term result of catheter ablation of idiopathic right ventricular tachycardia. Circulation 1990, 82:2093-9.

2 Klein LS, Shih HT, Hackelt FK, Zipes DP, Miles WM. Radiofrequency catheter ablation of ventricular tachycardia in patients with structural catheter ablation of ventricular tachycardia

3 Wilber DJ, Baerman J, Olshansky B, Kall J, Kopp D. Adenosine-sensitive ventricular tachycardia: clinical characteristics and response to catheter ventricular tachycardia: clinical chara

4 Calkins H, Kalbfleisch SJ, El-Atassi R, Langberg J, Morady F. Relation between efficacy of radiofrequency catheter ablation and site of origin of idiopathic ventricular tachycardia. Am $¥$ Cardiol 1993;71:827-33.

5 Nakagawa H, Beckman KJ, McClelland JH, et al. Radiofrequency catheter ablation of idiopathic left ventricular tachycardia guided by Purkinje potential. Circulation 1993;88:2607-17.

6 Wen MS, Yeh SJ, Wang CC, Lin FC, Chen IC, Wu D. Radiofrequency ablation therapy in idiopathic left ventricular tachycardia with no obvious structural heart disease. Circulation 1994;89:1690-6. 